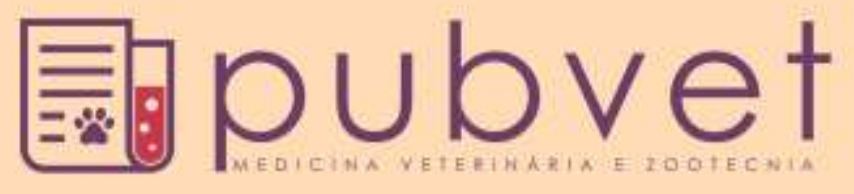

HTTP://DX.DOI.ORG/10.22256/PUBVET.VI1N2.199-205

\title{
Relação Programa Institucional de Bolsas de Iniciação à Docência PIBID-escola: aspectos relevantes para a melhoria da qualidade do ensino
}

\author{
Joniel Mendes de Araújo ${ }^{1}$, Johnny Iglesias Mendes Araujo², Ana Lucia Alves da Silva ${ }^{3}$, \\ Ranes Batista da Rocha ${ }^{4}$, Gilvan Rodrigues dos Santos ${ }^{5}$, Ryanne Fernandes de Oliveira ${ }^{6}$, \\ Geovania Figueiredo da Silva ${ }^{7}$, Luciana Barboza Silva ${ }^{8}$, Klendson Medeiros da Silva9 \\ ${ }^{I}$ Mestrando em Ciências Biológicas, Universidade Federal de Goiás, Samambaia, Brazil. E-mail: niel.ma30@gmail.com \\ ${ }^{2}$ Mestrando em Zootecnia, Universidade Federal do Piauí - Campus Prof. ${ }^{a}$ Cinobelina Elvas, E-mail: johnny-iglesias@hotmail.com \\ ${ }^{3}$ Especialista em Docência e Supervisão Escolar, Instituto de Ensino Superior Múltiplo. E-mail: anaufpi@hotmail.com \\ ${ }^{4}$ Liceciatura em Ciências Biológicas, Universidade Federal do Piauí - Campus Prof. ${ }^{a}$ Cinobelina Elvas, Bom Jesus, Piauí, Brasil. E-mail: \\ ranes.ufpi@hotmail.com \\ ${ }^{5}$ Mestrando em Ciências da Educação e Multidisciplinaridade, Faculdade Norte, E-mail: gr.seduc.pi@gmail.com \\ ${ }^{6}$ Graduanda em Fisioterapia - Centro Universitário Autônomo do Brasil, E-mail: ryannefernandes123@gmail.com \\ ${ }^{7}$ Esp. Gestão e Educação Ambiental, Instituto Nacional de Pós-Graduação e Ensino Superior, E-mail: geovaniafigueiredodasilva@ gmail.com \\ ${ }^{8}$ Dra. Entomologia Agrícola/Universidade Federal de Viçosa, E-mail: lubarbosabio@hotmail.com \\ ${ }^{9}$ Especialista em Redes de computadores Escola Superior Aberta Do Brasil, E-mail: klendsonmedeiros@gmail.com \\ *Autor para correspondência
}

RESUMO. A elaboração deste trabalho teve a proposta de analisar as contribuições do Programa Institucional de Bolsas e Iniciação à Docência (PIBID) para a melhoria da educação na Unidade Escolar Joaquim Parente na cidade de Bom Jesus - Piauí. A metodologia utilizada foi a observação participante no ambiente escolar junto à análise documental do Índice de Desenvolvimento da Educação Básica e do quadro de notas da escola. As contribuições que o PIBID proporcionou para a escola tem se efetivado por meio de seus três eixos: monitoria, aulas práticas e atividades complementares, que contribuíram para o processo de ensino-aprendizagem dos alunos, aumentando assim seu desempenho escolar e refletindo um aumento no Índice de Desenvolvimento da Educação Básica (IDEB) da escola.

Palavras chave: Bolsa PIBID, IDEB, qualidade de ensino.

\section{Relationship PIBID-school: relevant issues for improving the quality of education}

\begin{abstract}
The preparation of this work was the proposal to analyze the contributions of the Scholarship Program and Initiation to Teaching (PIBID) for the improvement of education in the School Unit Joaquim Parente in the town of Bom Jesus - Piauí. The methodology used was participant observation in the school environment with the documentary analysis of the Index of Basic Education Development and the bulletin board of the school. The contributions that PIBID provided to school has been effected by means of its three axes: monitoring, classes and complementary activities, which contributed to the teaching-learning process of the students, thus increasing their school performance and reflecting an increase in IDEB School.
\end{abstract}

Key words: PIBID, IDEB, teaching quality. 


\title{
Relación Programa Institucional de becas e Iniciación a la Docencia: PIBID-escuela aspectos relevantes para la mejoría de la calidad de la enseñanza
}

\begin{abstract}
RESUMEN. La elaboración de este trabajo tuvo la propuesta de analizar las contribuciones del Programa Institucional de Becas e Introducción a la docencia (PIBID) para la mejora de la educación en la unidad escolar Joaquim Parente en la ciudad de Bom Jesus - Piauí. La metodología utilizada fue la observación participante en el entorno escolar con el análisis de documentos del Índice de Desarrollo de la Educación Básica y el marco de notas escolares. Las contribuciones que PIBID proporcionó a la escuela se ha hecho efectiva a través de sus tres áreas: la supervisión, clases prácticas y actividades complementarias, lo que contribuyó al proceso de enseñanza y aprendizaje de los estudiantes, lo que aumentó su rendimiento escolar y que reflejó un aumento en el Índice de Desarrollo de la Educación Básica (IDEB) de la escuela.
\end{abstract}

Palabras clave: Becas PIBID, IDEB; calidad de enseñanza

\section{Introdução}

Atualmente a educação vive uma dicotomia, por um lado pode-se perceber a valorização da educação informal nos diversos fatores, e por outro lado à desvalorização da escola e dos profissionais da educação formal, os professores (Nadal, 2009). Graças a isso, o ensinar tem sofrido muito por não ser valorizado, o mesmo deve ser urgentemente modificado, pois atualmente com o grande avanço das tecnologias é preciso renovar, mudar e aperfeiçoar o ensino para que ele possa ser aplicado a todo e/ou qualquer aluno independentemente da classe em que este pertence.

Em busca da superação das suas dificuldades, a escola tenta romper com os paradigmas que as cerca até os dias atuais, que é a ideologia e a má organização do currículo escolar. Para que a escola se torne de fato democrática, como a mesma se diz ser, deve valorizar as ideias da sociedade na qual está inserida e buscar construir um currículo que atenda as reais necessidades dos alunos nos dias de hoje (Moreira, 2007).

O movimento de superação da crise escolar é ressaltado por Nadal (2009): que o papel da escola de reconfigurar seu Projeto Político Pedagógico, especificamente a reformulação de suas metas, objetivos, currículo, metodologia, recursos didáticos e gestão que acompanhem as mudanças e evoluções tecnológicas que o país está enfrentando. Afirma Libâneo (2007): a escola deverá estar aberta para acolher uma cultura crítica, onde abrange novas dimensões experienciais, afetivas e locais, rompendo os laços com a transmissão de conhecimentos clássicos da educação, esta precisa reelaborar o ensino e acompanhar as mudanças vivenciadas pela nova sociedade.

A ressignificação do conhecimento, não deve ser interpretado como desvalorização do saber didático, a escola deverá fazer a apropriação crítica do conhecimento, pois, ao preparar os alunos por meio da crítica, da compreensão das mudanças significativas do saber, o prepara de forma mais consciente para o novo disciplinamento (Veiga-Neto, 2007).

$\mathrm{Na}$ busca constante pela superação das dificuldades do ensino da rede pública e na necessidade de promover um avanço para a qualidade educacional, é que as universidades, em articulação com as escolas, usam seus programas para viabilizar $o$ processo de ensinoaprendizagem, ao mesmo tempo em que aperfeiçoa os acadêmicos dos cursos de licenciatura.

Um dos maiores desafios da educação é a formação de professores qualificados e comprometidos com as mudanças que acompanham o ensino (Feldmann, 2009). Por ser professor, sujeito mediador dos saberes, valores e atitudes, tem a maior responsabilidade de interpretar e reinterpretar o mundo junto à comunidade escolar, por isso, hoje a preocupação com a formação de um educador tem sido a alavanca para a criação de projetos que incentivem a docencia e a formação continuada (Feldmann, 2009).

O Programa Institucional de Bolsas de Iniciação à Docência (PIBID) é um programa instituído no âmbito do Ministério da Educação, da Fundação Coordenação de Aperfeiçoamento de Pessoal de nível Superior (CAPES) e do Fundo 
Nacional de Desenvolvimento da Educação (FNDE), que surgiu como uma nova proposta, que tem como um dos objetivos valorizar e incentivar o magistério e possibilitar aos acadêmicos dos cursos de licenciatura a participação em experiências metodológicas e práticas docentes inovadoras que serão desenvolvidas dentro das escolas públicas (CAPES, 2010).

A Lei de Diretrizes e Bases da Educação Nacional LDB nº 9394/96 em seu Título VI Dos Profissionais da Educação teve o parágrafo $5^{\circ}$ Incluído pela Lei $\mathrm{n}^{\circ} 12.796$, de 2013 que institui sobre o PIBID:

\section{$\S 5^{\circ}$ A União, o Distrito Federal, os Estados e os Municípios incentivarão a formação de profissionais do magistério para atuar na educação básica pública mediante programa institucional de bolsa de iniciação à docência a estudantes matriculados em cursos de licenciatura, de graduação plena, nas instituições de educação superior. (Incluído pela Lei $n^{o}$ 12.796, de 2013 - BRASIL, 2013)}

O PIBID, assim como vários outros projetos, estudos e pesquisas docentes no Brasil, está voltado para a busca de conhecimentos que priorize a relação entre a formação do professor versus sua prática pedagógica nas escolas brasileiras. Nessa perspectiva que Feldmann (2009) atenta para os três principais fatores que são tomados como essenciais para nortear os projetos de incentivo à docência: (1) valorização da experiência profissional como conhecimento; (2) articulação entre a teoria e prática; e (3) adequação do saber mediante o acesso das tecnologias informacionais e comunicacionais.

Esta pesquisa teve como intuito analisar as contribuições que foram propostas pelo PIBID, demonstradas através da execução do projeto de melhoria da qualidade da educação na Unidade Escolar Joaquim Parente na cidade de Bom Jesus - PI, melhorias como no processo de ensinoaprendizagem, nas aulas didáticas e principalmente nas aulas de reforços e práticas em laboratórios. O objeto de estudo foi às notas obtidas pelas avaliações realizadas pelo sistema de monitoramento do Instituto Nacional de Estudos e Pesquisas Educacionais Anísio Teixeira (INEP) ao demonstrar os efeitos de melhoria do desempenho escolar por meio dos indicadores do Índice de Desenvolvimento da Educação Básica (IDEB).

\section{Material e Métodos}

O presente trabalho foi realizado na cidade de Bom Jesus, no estado do Piaui. "Localizado "a uma latitude de $09^{\circ} 04^{\prime} 28^{\prime \prime}$ sul e a uma longitude de $44^{\circ} 21^{\prime} 31^{\prime \prime}$ oeste, estando a uma altitude de 277 metros, possui uma área de $5.469 \mathrm{~km}^{2}$.

Conforme o censo IBGE (2013), (Instituto Brasileiro de Geografia Estatística), 23.826 habitantes. Apresentando uma temperatura elevada, segundo o INMET (2010). (Instituto Nacional de Meteorologia), os termômetros às vezes ultrapassam os $40^{\circ} \mathrm{C}$. Os biomas predominantes neste local são: Cerrado e Caatinga. Segundo o IBGE (2008) Bom Jesus situa-se na Mesorregião sudoeste piauiense, microrregião Alto Médio do Gurguéia, Densidade 4,14 hab. $/ \mathrm{km}^{2}$, clima semiárido com distância até a capital de $635 \mathrm{~km}$. A cidade é referência no desenvolvimento do agronegócio, nos setores de comércio, indústria e serviços. Alem de congregar frequentemente habitantes de cidades circunvizinhas na resolução de negocios e estudantes.

A pesquisa foi realizada na Unidade Escolar Joaquim Parente, situada na cidade de Bom Jesus/PI, que atende 647 alunos, sendo 226 no Ensino Fundamental (matutino), 421 alunos estão cursando o Ensino Médio (diurno e noturno), dentre estes 29 alunos pertence à comunidade rural do Piripiri, 70 a do Eugenópolis e 32 alunos pertencem à comunidade do Corrente dos Matões, as localidades são interiores pertencentes ao Ensino Médio da escola.

A Universidade Federal do Piauí, credenciada pelo PIBID, contemplou a Unidade Escolar Joaquim Parente em 2010 com o programa PIBID com a proposta de formar uma parceria entre universidade e escola em prol de dois objetivos: incentivar a docência para os alunos do curso de Licenciatura em Ciências Biológicas e contribuir com a melhoria da qualidade da educação na escola pública.

A metodologia utilizada foi a de observação participante no ambiente escolar, análise documental do IDEB e análise do quadro de notas da escola. Dentro dessa perspectiva a intenção da pesquisa foi realizar uma investigação do cotidiano escolar com o objetivo de perceber as contribuições que o PIBID pode fornecer para a melhoria do desempenho escolar dos alunos durantes esses dois anos. Desse modo, seguiu-se a utilização de um dos principais instrumentos 
básicos para a investigação daquele cotidiano: observação participante (Bogdan \& Biklen, 1997 apud Taveira, 2008).

Durante a observação, é importante ressaltar que a proposta foi a de analisar todas as relações que envolviam os alunos no decorrer do processo de ensino aprendizagem, e a maneira como o PIBID utilizava sua prática docente para intervir nesse processo de assimilação de conteúdos teóricos. A esse princípio, não foi permitido que dirigíssemos o olhar a pontos fixos, e nem deixar transparecer ações esquematizadas, como sugere Taveira (2008) é necessário chegar à escola com sentidos todos alertas, para que a análise não seja apenas daquilo esperado, mas de tudo que a escola oferece.

\section{Resultado e discussão}

$\mathrm{Na}$ análise dos dados foi observada significativa melhoria do desempenho escolar, considerado como consequência das contribuições do PIBID, para o processo de ensino aprendizagem dos alunos.

Conforme planilha 1 , no primeiro ano da avaliação do IDEB em 2007, a escola Joaquim Parente obteve nota de 2,5, em 2009 a meta esperada era de 2,6, porém a escola obteve 1,7. Após a nova análise de 2011, onde já havia o PIBID na escola percebe-se que o IDEB aumentou, pois, a meta esperada para 2011 era de 2,8 e a escola obteve média de 3,1 (INEP, 2012).

Ficou demonstrado que as atividades desenvolvidas pelo PIBID contribuíram para a melhoria da qualidade da educação nessa escola. Analisando todas as demais escolas referentes aos outros municípios sendo uma federal o Colégio Agrícola de Bom Jesus e as demais sendo estaduais, verifica-se, portanto que a média das notas tem aumentado, mas a da Unidade Escolar Joaquim Parente foi bem maior que a projeção esperada para o ano de 2011 que era de 2,8 e obteve 3,1 .

Planilha 1. Inep 2012 divulgado pela $14^{\text {a }}$ Gerência Regional de Educação de Bom Jesus - PI

\begin{tabular}{|c|c|c|c|c|c|c|c|c|}
\hline \multirow{2}{*}{ Município } & \multirow{2}{*}{ Nome da Escola } & \multirow{2}{*}{$\begin{array}{c}\text { IDEB } 2005 \\
(\mathrm{~N} \times \mathrm{P})\end{array}$} & \multirow{2}{*}{$\begin{array}{c}\text { IDEB } 2007 \\
(\mathrm{~N} \times \mathrm{P})\end{array}$} & \multirow{2}{*}{$\begin{array}{c}\text { IDEB } 2009 \\
(\mathrm{~N} \times \mathrm{P})\end{array}$} & \multirow{2}{*}{$\begin{array}{c}\text { IDEB } 2011 \\
(\mathrm{~N} \times \mathrm{P})\end{array}$} & \multicolumn{3}{|c|}{ Projeções } \\
\hline & & & & & & 2007 & 2009 & 2011 \\
\hline Bom Jesus & Colégio Agrícola de Bom Jesus & - & 4,3 & 6,3 & - & - & 4,4 & 4,6 \\
\hline Bom Jesus & \begin{tabular}{|l} 
Centro de Ensino Médio de \\
Tempo Integral - Franklin Dória
\end{tabular} & 3,5 & 2,9 & 3,4 & - & 3,5 & 3,7 & 4 \\
\hline Eliseu Martins & $\begin{array}{l}\begin{array}{l}\text { Ginásio Nossa Senhora de } \\
\text { Fátima }\end{array} \\
\end{array}$ & - & - & - & - & - & - & - \\
\hline Cristino Castro & $\begin{array}{l}\text { Centro Educacional Integrado } \\
\text { ADENAUER }\end{array}$ & 2,2 & 3,1 & 3,8 & 4 & 2,3 & 2,7 & 3,2 \\
\hline Manoel Emídio & $\begin{array}{l}\text { Unidade Escolar Antônio Borges } \\
\text { Leal }\end{array}$ & 3,1 & 3,2 & 4,7 & 3,9 & 3,2 & 3,3 & 3,6 \\
\hline Santa Luz & $\begin{array}{l}\text { Unidade Escolar Professora Iraci } \\
\text { Barros Pinto }\end{array}$ & - & 3,8 & 3,2 & 3,7 & - & 3,9 & 4,1 \\
\hline $\begin{array}{l}\text { Redenção do } \\
\text { Gurguéia }\end{array}$ & $\begin{array}{l}\text { Unidade Escolar José Dário dos } \\
\text { Santos }\end{array}$ & 2,6 & 3,6 & 3,4 & 3,6 & 2,6 & 2,7 & 3 \\
\hline $\begin{array}{l}\text { Alvorada do } \\
\text { Gurguéia }\end{array}$ & Escola Agrícola do DNOCS & - & 3,5 & 3,6 & 3,5 & - & 3,6 & 3,8 \\
\hline Bom Jesus & $\begin{array}{l}\begin{array}{l}\text { Unidade } \\
\text { Parente }\end{array} \\
\end{array}$ & - & 2,5 & 1,7 & 3,1 & - & 2,6 & 2,8 \\
\hline Bom Jesus & Unidade Escolar Araci Lustosa & - & - & - & 2,9 & - & - & - \\
\hline Cristino Castro & $\begin{array}{l}\text { Unidade Escolar Francisco de } \\
\text { Miranda }\end{array}$ & - & - & - & 2,9 & - & - & - \\
\hline Bom Jesus & $\begin{array}{l}\text { Unidade Escolar José Lustosa } \\
\text { Elvas Filho }\end{array}$ & - & - & - & 2,2 & - & - & - \\
\hline
\end{tabular}

As perspectivas acerca da melhoria da educação na escola Joaquim Parente ainda são muitas e toda a comunidade educacional da escola está trabalhando em prol desse desenvolvimento, rumo ao alcance da meta estabelecida pelo
Ministério da Educação e Cultura (MEC), que é a nota 6,0. As contribuições que o PIBID proporcionou para a escola foi efetivado por meio de seus três eixos: monitoria, aulas práticas e atividades complementares. Atividades estas que 
são realizadas frequentemente, de modo a contribuir para o aperfeiçoamento do conhecimento dos alunos, aumentando o seu desempenho escolar e refletindo um aumento no IDEB da escola.

As atividades de monitoria ocorrem conforme a necessidade dos alunos, sendo ela realizada de forma individual, onde o aluno que tem dúvidas sobre algum assunto voltado a área de ciências ou biologia, recorre a um bolsista do PIBID para auxiliar em sua dificuldade. Quando a monitoria é de classe, o bolsista faz o reforço com a turma toda ou com uma maior porcentagem dos alunos da sala, sobre assuntos que os alunos estão com dificuldades de aprendizagem ou até mesmo assuntos interdisciplinares, assim como sugeridos nos Parâmetros curriculares Nacionais (PCNs). A monitoria resolveu várias questões referente ao déficit qualitativo dos alunos. A monitoria se revelou uma forma diferente do aluno assimilar o conhecimento que não foi efetivado em sala de aula com o professor.

As aulas práticas é um dos eixos do PIBID e pretende desenvolver o lado cognitivo dos alunos, no momento que a existe a relação entre a teoria e a prática ocorre uma maior assimilação dos conteúdos didáticos e também um melhor rendimento acadêmico dos educandos. Krasilchik (2008), atenta para um ensino articulado, onde a prática não seja apenas uma alusão distante da teoria, mas, que esta, a prática, seja companheira árdua no decorrer do processo ensinoaprendizagem, para que os alunos não só assimilem o conteúdo, mas, também possam agir de forma crítica sobre os processos da natureza bem como resolver problemas cotidianos que envolvam as ciências.

Graças a essa forma de ensino, com a ajuda dos Pibidianos, houve aulas de campos com os alunos da última série do Ensino Médio que consistiu em levar os alunos da turma do $3^{\circ}$ ano do turno tarde/noite, para uma aula de campo na Estação Ecológica de Uruçuí-Una que está localizada no sul do estado do Piauí, no município de Baixa Grande do Ribeiro, desmembrado de Ribeiro Gonçalves, entre os rios Uruçuí-Una e Riozinho. Nessa ocasião foram realizadas atividades para despertar o desenvolvimento cognitivo e a interação aluno/ambiente, com a perspectiva de estimular o conhecimento ecológico dos alunos e mobilizar para as questões de cunho ambiental. Com isso, os resultados das atividades realizadas durante a aula de campo foram bastante positivos, pois, foi mediante a relação teórico/prático dos conteúdos da disciplina de Biologia, que os estudantes puderam entender conceitos ecológicos e medidas preventivas sobre impactos ambientais na região de estudo. Sobre esse efeito percebe-se que houve uma ressignificação no conhecimento dos alunos envolvidos no projeto e se sensibilizaram com questões de cunho ecológico e da preservação ambiental. Além disso, o desenvolvimento das atividades promoveu $\mathrm{o}$ estimulo e o senso crítico dos alunos participantes da aula de campo e simultaneamente melhorou seu rendimento escolar.

No eixo pedagógico do PIBID, o grupo realizou projetos de intervenção escolar sob o contexto dos Parâmetros Curriculares Nacionais (PCNs) e de forma interdisciplinar, onde envolvem assuntos referentes ao Meio Ambiente, Saúde, Trabalho e Tecnologias. Nesse eixo os alunos tiveram a oportunidade de trabalhar em equipe pela melhoria do ambiente escolar e da sociedade em geral. Os temas são quase sempre definidos com auxílio de toda comunidade escolar e sobre fatores emergentes, por exemplo: campanhas contra dengue, lixo, riacho grotão e dentre vários outros problemas que estão presentes na comunidade onde a escola está inserida.

$\mathrm{O}$ grupo PIBID reconhece à importância de se trabalhar a interdisciplinaridade, como forma de proporcionar aos alunos, a formação de conhecimentos sólidos e também promover o desenvolvimento de habilidades que serão aplicadas em seu futuro exercício profissionais.

A proposta interdisciplinar de ensino pode ser concretizada basicamente sobre dois aspectos. A partir de uma abordagem que privilegie a compreensão do processo de produção do conhecimento, ou, o que é mais comum, a partir de um tema gerador único que irá ser trabalhado pelas diferentes disciplinas.

Uma das possibilidades de se trabalhar a interdisciplinaridade, executadas no PIBID, são os projetos, por meio dos quais, os professores podem introduzir o estudo de temas que não pertencem a uma disciplina específica, mas que envolvam duas ou mais disciplinas. Tais projetos didáticos são importantes, pois abrem novas possibilidades de aprendizagem aos estudantes: viver situações em que é necessário tomar uma decisão sobre o caminho a seguir; se convencer da necessidade de elaborar um cronograma, considerar uma meta e diagnosticar as condições iniciais para realizar o projeto; decidir que estudos 
devem ser realizados para resolver um problema; entre outros. A interdisciplinaridade é uma discussão emergente no meio educacional: uma forma de se pensar no interior da Educação, enfim, é a superação da abordagem disciplinar, tradicionalmente fragmentária. Essa, frequentemente, é apontada como incapaz de atender às demandas por um ensino contextualizado. Embora esse enfoque não seja recente, as discussões sobre o tema no Brasil ocorrem desde a década de 1970 (Fazenda, 2002), e apenas agora têm encontrado terreno fértil para se propagar, em virtude de estarem presentes nos parâmetros oficiais, que norteiam a prática educacional, e no discurso de professores, coordenadores e administradores do ensino.

Graças ainda às contribuições do PIBID, pode se perceber por meio da planilha 2 que mostra além dos dados destacados anteriormente, a porcentagem do aumento das médias de crescimento da nota do IDEB de cada escola, o quanto a Unidade Escolar Marcos Parente superou as projeções e cresceu e está crescendo com a ajuda do PIBID.

O PIBID se tornou um projeto efetivo na escola Joaquim Parente com a proposta de continuar contribuindo para a efetivação da qualidade e melhoria do ambiente escolar e cotidianamente proporcionar meios que facilitem a aprendizagem e desenvolvimento dos alunos como um todo. E nessa perspectiva de melhoria da educação o PIBID também incentiva a construção da identidade do futuro profissional docente e estes graduandos de licenciatura percebem na prática a realidade vivenciada pelas escolas públicas, e contribuem para a as mudanças.

Planilha 2. Inep 2012 divulgado pela $14^{a}$ Gerência Regional de Educação de Bom Jesus - PI

\begin{tabular}{|c|c|c|c|c|c|c|c|c|c|}
\hline \multirow[b]{2}{*}{ Município } & \multirow[b]{2}{*}{ Nome da Escola } & \multirow{2}{*}{$\begin{array}{c}\text { IDEB } \\
2005(\mathrm{~N} \mathrm{x} \\
\mathrm{P}) \\
\end{array}$} & \multirow{2}{*}{$\begin{array}{c}\text { IDEB } \\
2007(\mathrm{~N} \mathrm{x} \\
\mathrm{P}) \\
\end{array}$} & \multirow{2}{*}{$\begin{array}{c}\text { IDEB } \\
2009(\mathrm{~N} \mathrm{x} \\
\mathrm{P}) \\
\end{array}$} & \multirow{2}{*}{$\begin{array}{c}\text { IDEB } \\
2011(\mathrm{~N} \mathrm{x} \\
\mathrm{P}) \\
\end{array}$} & \multicolumn{3}{|c|}{ Projeções } & \multirow{2}{*}{$\begin{array}{l}\% \text { de } \\
2009- \\
2011 \\
\end{array}$} \\
\hline & & & & & & 2007 & 2009 & 2011 & \\
\hline Bom Jesus & $\begin{array}{l}\text { Colégio Agrícola de Bom } \\
\text { Jesus }\end{array}$ & - & 4,3 & 6,3 & - & - & 4,4 & 4,6 & \\
\hline Bom Jesus & \begin{tabular}{|l|} 
Centro de Ensino Médio de \\
Tempo Integral - Franklin \\
Dória
\end{tabular} & 3,5 & 2,9 & 3,4 & - & 3,5 & 3,7 & 4 & \\
\hline Eliseu Martins & $\begin{array}{l}\text { Ginásio Nossa Senhora de } \\
\text { Fátima }\end{array}$ & - & - & - & - & - & - & - & \\
\hline $\begin{array}{l}\text { Cristino } \\
\text { Castro }\end{array}$ & 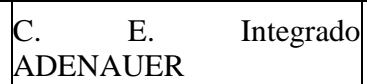 & 2,2 & 3,1 & 3,8 & 4 & 2,3 & 2,7 & 3,2 & - \\
\hline $\begin{array}{l}\text { Manoel } \\
\text { Emídio } \\
\end{array}$ & $\begin{array}{l}\text { Unidade Escolar Antônio } \\
\text { Borges Leal }\end{array}$ & 3,1 & 3,2 & 4,7 & 3,9 & 3,2 & 3,3 & 3,6 & - \\
\hline Santa Luz & $\begin{array}{l}\text { U. E. Professora Iraci } \\
\text { Barros Pinto }\end{array}$ & - & 3,8 & 3,2 & 3,7 & - & 3,9 & 4,1 & - \\
\hline $\begin{array}{l}\text { Redenção do } \\
\text { Gurguéia }\end{array}$ & U. E. José Dário dos Santos & 2,6 & 3,6 & 3,4 & 3,6 & 2,6 & 2,7 & 3 & - \\
\hline $\begin{array}{l}\text { Alvorada do } \\
\text { Gurguéia }\end{array}$ & E. Agrícola do DNOCS & - & 3,5 & 3,6 & 3,5 & - & 3,6 & 3,8 & - \\
\hline Bom Jesus & U. E. Joaquim Parente & - & 2,5 & 1,7 & 3,1 & - & 2,6 & 2,8 & 82,4 \\
\hline Bom Jesus & U. E. Araci Lustosa & - & - & - & 2,9 & - & - & - & 15,6 \\
\hline $\begin{array}{l}\text { Cristino } \\
\text { Castro }\end{array}$ & $\begin{array}{l}\text { Unidade Escolar Francisco } \\
\text { de Miranda }\end{array}$ & - & - & - & 2,9 & - & - & - & 5,9 \\
\hline Bom Jesus & $\begin{array}{l}\text { Unidade Escolar José } \\
\text { Lustosa Elvas Filho }\end{array}$ & - & - & - & 2,2 & - & - & - & 5,3 \\
\hline
\end{tabular}

O PIBID, nesse aspecto, pode ser considerado no cenário municipal, como o aplicador de um novo paradigma educacional, desperta para a necessidade de romper com essa visão fragmentária, e apresenta a proposta de uma ação pedagógica mais crítica, sistematizada, e, que atenda os anseios socioculturais dos atores envolvidos no processo educacional.
Outra meta alcançada foi, a elevação das notas na turma, o que evidencia que a busca por novas metodologias de ensino proporciona, não só aos alunos, mas também aos professores, estagiários e Pibidianos, uma maior assimilação dos conteúdos e a aquisição de uma prática reflexiva por parte dos docentes e integrantes do PIBID. 
Quando se trata de, procurar uma metodologia para se trabalhar de forma interdisciplinar a proposta de metodologias que promovam uma maior interação entre os alunos e, entre os alunos e o professor deve ser priorizado.

Assim, a simulação de um ambiente de sala de aula utilizando metodologias e recursos didáticos variados proporciona aos alunos um ambiente propício ao desenvolvimento cognitivo e de habilidades inerentes à prática de pesquisa sistemática (busca de referências para estudo, observação criteriosa, comparação de resultados, composição de textos, etc.) e aos Pibidianos excelentes oportunidades de aperfeiçoamento docente.

\section{Conclusão}

No contexto do programa PIBID existem atividades que elevam o saber cognitivo dos alunos, desde monitoria individual e de classe, aulas práticas e de campo, e participação em projetos de intervenção pedagógica de cunho multidisciplinar. O PIBID comprovou que teve imensa contribuição para o aumento do IDEB da escola não só no quantitativo das notas, mas também por ter propiciado um ambiente de maior interação e de respeito mútuo entre toda comunidade escolar e a partir do momento em que estes alunos questionam a seus próprios atos, contribuí, portanto para o seu próprio desenvolvimento, adquirindo novos conhecimentos e despertando o seu pensamento crítico. Sob o ponto de vista da importância da das aulas de campo que o PIBID pode promover, é certo que o mesmo pode despertar uma sensibilização por parte dos alunos, para problemas ambientais e consequente conscientização. E graças a isso, como futuros cidadãos os mesmos poderão intervir de forma direta no meio ambiente, com ações que visam à proteção, conservação e equilíbrio dos impactos ambientais.

Em relação à atribuição do PIBID quanto à complexidade ambiental, a mesma exige da interdisciplinaridade a contribuição de novos conhecimentos e práticas de pesquisas, intervindo e colaborando para geração de algo cientificamente novo, possibilitando melhorias concretas para tantos problemas encontrados na sociedade. Os desafios percebidos são grandes, existem muitas barreiras, mas existem também os meios de vencer. Com a pretensão de implantar novos métodos de ensino, objetivando a formação de sujeitos críticos, que interfiram na realidade sociocultural, que neste projeto foi adotado e o resultado auferido é a de que a interdisciplinaridade se apresenta como uma ótima opção.

\section{Referências Bibliográficas}

CAPES. Programa Institucional de Bolsas de Iniciação à Docência. 2010 [online]. Home Page: http://www.capes.gov.br/educacaobasica/capesPIBID Acesso em dezembro de 2012.

Feldmann, M. G. 2009. Formação de professores e escola na contemporaneidade. - São Paulo: Editora Senac São Paulo.

IBGE. Instituto Brasileiro de Geografia e estatística. Geografia (2008). Disponível em: http://www.ibge.gov.br/home/mapa_site/mapa site.php\#economia. Acesso: 20 de junho de 2014.

IBGE. Instituto Brasileiro de Geografia e estatística. Perfil dos Municípios Brasileiros (2013). Disponível em: http://www.ibge.gov.br/home/estatistica/econ omia/perfilmunic/2013/. Acesso: 20 de junho de 2014.

INEP. Instituto Nacional de Pesquisas Educacionais Anísio Teixeira. Sinopse Estatística da Educação Superior (2012). Disponível em: www.inep.gov.br. Acesso em agosto de 2014.

INMET. Instituto Nacional de Meteorologia. Anomalias de Precipitação (2010). Disponível em:

http://www.inmet.gov.br/html/clima.php?lnk= /html/clima/anomalias/index.html. Acesso em julho de 2014.

Krasilchik, M. 2008. Prática de Ensino de Biologia. São Paulo: EDUSP, Ed.

Libâneo, J. C. 2007. A escola com que sonhamos é aquela que assegura a todos a formação cultural e científica para a vida pessoal, profissional e cidadã. In: Costa, M. V. A escola tem futuro? $2^{\mathrm{a}}$.ed. Rio de Janeiro: Lamparina, 23-50.

Moreira, A. F. B. 2007. A escola poderia avançar um pouco no sentido de melhorar a dor de tanta gente. In: Costa, M. V. A escola tem futuro? 2. ed. Rio de Janeiro: Lamparina, 51-76. 
Nadal, B. G. 2009. A escola e sua função social: uma compreensão à luz do projeto da modernidade. In: Feldmn, M. G. Formação de professores e escola na contemporaneidade. São Paulo: Editora Senac São Paulo, 19-34.

Taveira, E. B. 2008. A pesquisa dos/nos/com os cotidianos e suas múltiplas possibilidades de apresentação. In: Oliveira, I. B. \& Alves, N. Pesquisa nos/dos/com os cotidianos das escolas. Sobre redes de saberes. $3^{\mathrm{a}}$ ed. Rio de Janeiro: DP\&A, 119-139.

Veiga-Neto, A. 2007. Pensar a escola como uma instituição que pelo menos garanta a manutenção das conquistas fundamentais da modernidade. In: Costa, M. V. A escola tem futuro? $2^{\mathrm{a}}$.ed. Rio de Janeiro: Lamparina, 103126.

\section{Article History:}

Received 3 November 2016

Accepted 25 November 2016

Available on line 1 February 2017

License information: This is an open-access article distributed under the terms of the Creative Commons Attribution License 4.0, which permits unrestricted use, distribution, and reproduction in any medium, provided the original work is properly cited 